\title{
Lung ultrasonography for early management of patients with respiratory symptoms during COVID-19 pandemic
}

\author{
Andrea Smargiassi ${ }^{1}$ (1) Gino Soldati ${ }^{2} \cdot$ Alberto Borghetti $^{3}$ - Giancarlo Scoppettuolo ${ }^{3}$ - Enrica Tamburrini ${ }^{3}$. \\ Antonia Carla Testa $^{4,5}$. Francesca Moro ${ }^{4}$. Luigi Natale ${ }^{6,7}$. Anna Rita Larici ${ }^{6,7}$. Danilo Buonsenso ${ }^{8}$. Piero Valentini ${ }^{8}$. \\ Gaetano Draisci ${ }^{9}$ Bruno Antonio Zanfini ${ }^{9}$. Maurizio Pompili ${ }^{10}$. Giovanni Scambia ${ }^{4,5}$. Antonio Lanzone ${ }^{4,5}$. \\ Francesco Franceschi ${ }^{11}$. Gian Ludovico Rapaccini ${ }^{10}$ - Antonio Gasbarrini ${ }^{10}$. Paolo Giorgini ${ }^{12}$. Luca Richeldi ${ }^{1,13}$. \\ Libertario Demi ${ }^{12}$ (1) Riccardo Inchingolo ${ }^{1}$ (1)
}

Received: 18 May 2020 / Accepted: 23 June 2020 / Published online: 7 July 2020

(c) Società Italiana di Ultrasonologia in Medicina e Biologia (SIUMB) 2020

\begin{abstract}
COVID-19 pandemic is representing a serious challenge to worldwide public health. Lung Ultrasonography (LUS) has been signaled as a potential useful tool in this pandemic contest either to intercept viral pneumonia or to foster alternative paths. LUS could be useful in determining early lung involvement suggestive or not of COVID-19 pneumonia and potentially plays a role in managing decisions for hospitalization in isolation or admission in general ward. In order to face pandemic, in a period in which a large number of emergency room accesses with suspicious symptoms are expected, physicians need a standardized ultrasonographic approach, fast educational processes in order to be able to recognize both suggestive and not suggestive echographic signs and shared algorithms for LUS role in early management of patients.
\end{abstract}

Keywords Lung Ultrasound · COVID-19 · Sonographic interstitial syndrome

Riccardo Inchingolo

riccardo.inchingolo@policlinicogemelli.it

1 Pulmonary Medicine Unit, Department of Medical and Surgical Sciences, Fondazione Policlinico Universitario A. Gemelli IRCCS, Largo Gemelli, 8, 00168 Rome, Italy

2 Diagnostic and Interventional Ultrasound Unit, Valle del Serchio General Hospital, Lucca, Italy

3 UOC Malattie Infettive, Dipartimento Scienze di Laboratorio e Infettivologiche, Fondazione Policlinico Universitario A. Gemelli IRCCS, Rome, Italy

4 Dipartimento Scienze della Salute della Donna, del Bambino e di Sanità Pubblica, Fondazione Policlinico Universitario A. Gemelli IRCCS, Rome, Italy

5 Dipartimento Scienze della Vita e Sanità Pubblica, Università Cattolica del Sacro Cuore, Rome, Italy

6 Department of Diagnostic Imaging, Oncological Radiotherapy and Hematology, Fondazione Policlinico Universitario A. Gemelli IRCCS, 00168 Rome, Italy

7 University Department of Radiological and Hematological Sciences, Section of Radiology, Università Cattolica del Sacro Cuore, 00168 Rome, Italy
8 Paediatric Unit, Department of Woman and Child Health and Public Health, Fondazione Policlinico Universitario A. Gemelli IRCCS, Rome, Italy

9 Science of Emergency, Anesthesiology, Intensive Care Therapy Fondazione Policlinico Universitario A Gemelli IRCCS, Rome, Italy

10 Department of Medical and Surgical Sciences, Fondazione Policlinico Universitario A. Gemelli IRCCS, Largo Gemelli, 8, 00168 Rome, Italy

11 Emergency Medicine, Fondazione Policlinico Universitario A. Gemelli IRCCS, Rome, Italy

12 Department of Information Engineering and Computer Science, Ultrasound Laboratory Trento, University of Trento, Trento, Italy

13 Dipartimento di Scienze Cardiovascolari e Toraciche, Università Cattolica del Sacro Cuore, 00168 Rome, Italy 


\section{Introduction}

The recent pandemic caused by Sars-Cov-2, spreading from Wuhan, China, in December 2019 is representing a serious challenge to worldwide public health and healthcare structures. The global emergency needs a unified approach to better early manage patients when, in a pandemic context, a large number of emergency room accesses are expected with a symptomatology characterized by cough, dyspnea and fever [1, 2]. Novel coronavirus disease (COVID-19) has a very heterogeneous clinical behaviour ranging from asymptomatic cases, different degrees of flu-like symptomatology until to cases of pneumonia with possible evolution into severe respiratory failure [3, 4].

Lung ultrasonography (LUS) has been signaled as a potential useful tool in this pandemic context in which the common and dangerous complication of COVID-19 symptomatology is represented by viral pneumonia [5, 6].

Viral pneumonia by Sars-Cov-2 is characterized by alveolar exudation and damage, interstitial thickening with patchy distribution with centrifugal extension and subpleural involvement. In advanced phases also consolidations and ARDS histologic pattern are observed [7]. Coagulation disorders and embolic/ischemic damages on lungs may explain, at least in part, the origin of some subpleural consolidations and a severe progression of respiratory failure [8, 9].

One of the characteristics of LUS is to intercept alterations affecting tissue/air content in peripheral lung parenchyma [10-13].

Normally, peripheral airspace geometry of lung hampers ultrasound incident waves thus determining a complete back reflection. In this healthy contest ultrasound (US) images are characterized by horizontal artifacts beyond the pleural plane [10, 12].

When peripheral airspace (PAS) geometry of lung is subverted from any cause (tissue/air ratio is reduced), ultrasound incident waves, in relations to their wavelength, could enter acoustic channels and be trapped in acoustic microholes on pleural plane. Vertical artifacts are visible in US images, resulting in the so-called Sonographic Interstitial Syndrome (SIS) indicative of a hyperdense preconsolidated state of lungs [10-16].

Moreover, it has been described how some characteristics of SIS could be indicative either of pneumogenic primary pathology or secondary involvement, like in case of cardiogenic pulmonary edema.

Therefore, LUS could be useful, being performed during diagnostic processes, in determining early lung involvement and potentially playing a role in managing decisions both intercepting cases of suspected COVID-19 pneumonia and suggesting alternative diagnosis. This could foster and speed up hospitalization in general ward when applicable.
A standardized ultrasonographic approach and fast educational processes are needed to have most of physicians able to recognize LUS signs and to build shared algorithms for LUS role in early management of patients.

\section{Standardized ultrasonographic approach}

In our experience in performing LUS in suspected COVID19 patients, both pocket and wireless devices [17] than standard ultrasound machines can be used better if dedicated exclusively to COVID-19 patients to reduce healthcare operator/patients infection. In any case maximum care for sterilization is necessary following last recommendations [18].

LUS can be performed using a standardized acquisition protocol with standardized setting conditions: using low MI, avoiding harmonic images and cosmetic filters, keeping one focal point on pleural line, avoiding saturation phenomena of pleural line and using the highest frame rate possible.

For patients able to maintain the sitting position a standard sequence of 14 evaluations (three posterior, two lateral and two anterior for each hemithorax) has been proposed in peculiar anatomic landmarks using a progressive numbering starting from right posterior basal regions. A modified acquisition protocol has been proposed in case of patients that are not able to keep the sitting position such as in critical care settings [17].

In these cases, it might be difficult to evaluate the posterior areas, currently considered a "hot-area" for COVID-19 pneumonia. Operators should try to have a partial view of the posterior basal areas by moving patients on both lateral sides and then, continue echographic assessment from right basal region on mid-axillary line below the internipple line in supine position [17].

A scoring system of LUS findings in COVID-19 patients has been proposed, ranging from 0 to $3[6,17]$. This scoring system is based on characteristics of pleural line and vertical artifacts.

Score 0: pleural line is regular. Horizontal reverberant artifacts and mirror effects are present.

Score 1: pleural line has slight alterations with sporadic vertical artifacts due to alteration of PAS geometry.

Score 2: pleural line has relevant alterations. Progression of subversion of PAS geometry causes a predominance of vertical artifacts. Small subpleural consolidations can be present.

Score 3: pleural line is irregular and cobbled. White lung with or without larger consolidations may be present.

\section{Educational programs}

In order to exploit LUS as useful tool to face COVID 19 viral pneumonia, hospitals need to have most of physicians 
involved in COVID 19 management able to recognize US suspicious patterns. Short educational programs should be performed to train physicians to standardize acquisition protocols and to recognize US patterns in patients with symptoms compatible with viral pneumonia.

Features that need to be identified are $[5,10,12,14]$ :

Characteristics of pleural line: normally the pleural line is regular and smooth. The same in case of cardiogenic pulmonary edema, especially in early interstitial phase. Pneumogenic pathological findings are irregularities and coarse-cobbled aspect.

Horizontal artifacts beyond the pleural line: conventionally called A-lines representing reverberant artifacts of pleural line. Mirror effects of acoustic interfaces of structures of chest wall can be present between reverberations of pleural lines. Mirror effects and reverberant artifacts of pleural line are associated to normal disposition of peripheral airspace geometry in inflated lungs.

Vertical artifacts beyond the pleural plane: conventionally called B-lines representing the openings of acoustic channels (shrunk and folded alveoli, water, tissue, fluids) on pleural plane due to alterations of peripheral airspace geometry by pathological conditions.

White lung: completely white artifactual field beyond the pleural plane without horizontal phenomena. Severe alterations of peripheral airspace geometry, with small airspaces randomly distributed.

Sonographic interstitial syndrome (SIS): the presence of vertical artifacts or/and white lung in echographic scans.

It is indicative of hyperdense pre-consolidated state of lung on its parenchymal periphery.

Lung consolidations: multi-shaped hypoechoic areas emerging on pleural plane.

Pleural effusions: intrathoracic fluid collection.

The presence of alterations of pleural line and SIS with patchy bilateral distribution in a symptomatic context compatible with COVID-19 is a suspicious US pattern [5]; similarly, white lung with bilateral patchy distribution associated or not with small subpleural consolidations, in the same symptomatic context is highly suggestive of COVID-19 pneumonia, but not definitively specific (Fig. 1) [5, 19, 20].

Sonographic interstitial syndrome with a homogeneous bilateral distribution without spared areas, pleural line smooth and regular, bright vertical artifacts with gravitational distribution, is suggestive of secondary involvement of lungs in cardiogenic pulmonary edema.

Moreover, large unilateral consolidations usually are not a compatible pattern as well as large pleural effusions [5].

Pneumologists, infectiologists, internists, emergency room doctors, radiologists, obstetricians/gynecologists and pediatricians might be potential targets of these educational

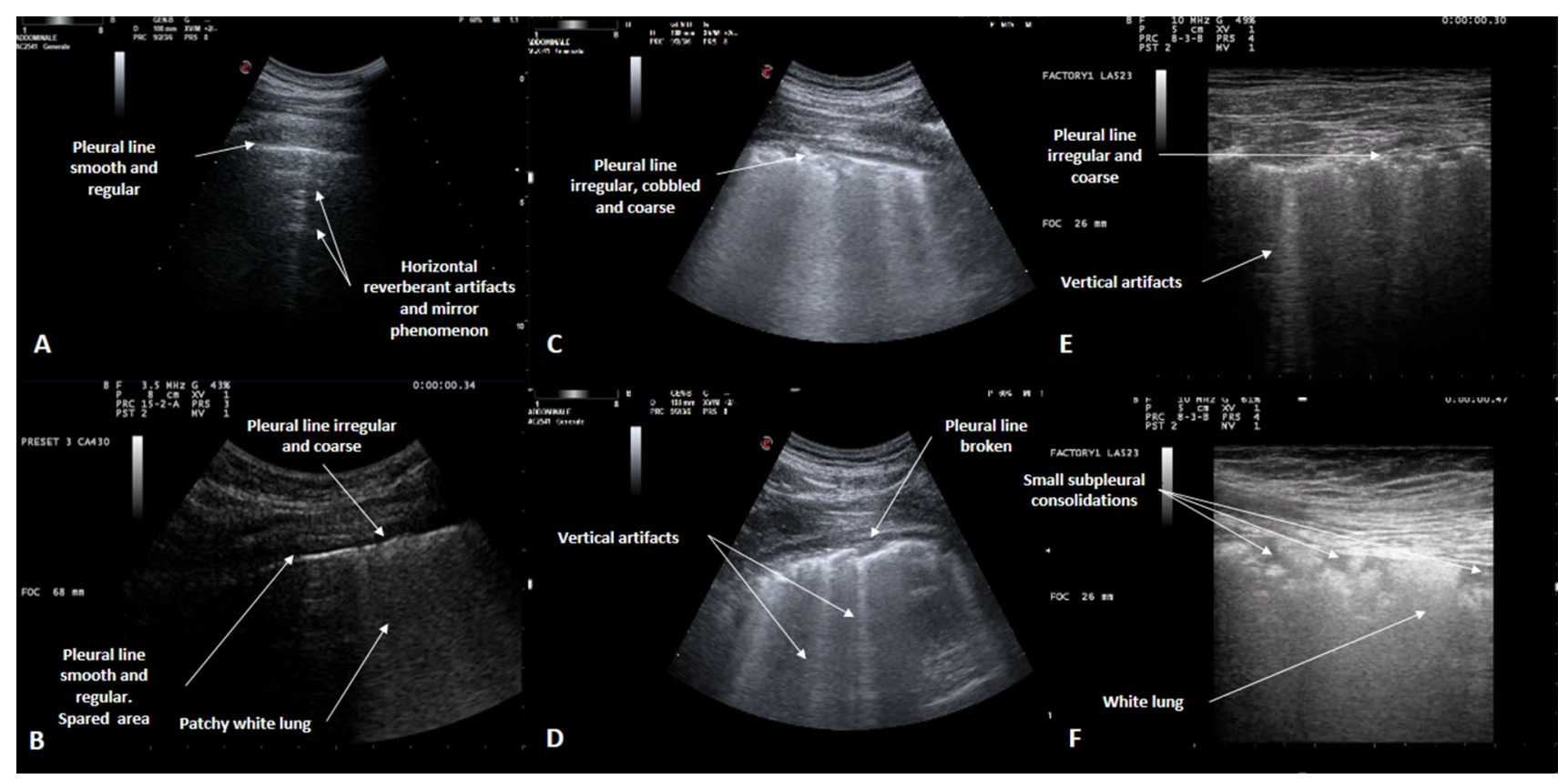

Fig. 1 LUS features that can be detected in patients with symptomatology compatible with COVID-19 and may suggest pulmonary involvement in the pandemic context. a, b Pleural line smooth and regular with horizontal reverberant artifacts and mirror phenomenon: spared area. b-f Pleural line irregularities (broken, coarse and cob- bled) c-e Vertical artifacts: openings of acoustic channels due to alterations of peripheral airspace geometry by pathological conditions. b Patchy white lung (f) white lung with small subpleural consolidations 
programs. Some of these specialists usually are already sonographers with long-time experience.

In our hospital 60 physicians of previously mentioned medical specialties, have been trained with a $2 \mathrm{~h}$ lesson, tailored on acquisition protocol and echographic patterns.

Experiences of application of this program, in obstetricians/gynecological area, have been published [21, 22].

\section{LUS and clinical management of patients with suspected symptoms}

LUS can have a pivotal role in early management of patients admitted to the emergency room with aspecific respiratory symptoms, but suspected of COVID-19 in pandemic contest (fever, dry cough and dyspnea). Patients should undergo LUS to better define subsequent management paths.

In fact, in these patients, whatever will be the results of a Chest X-ray (CXR), if performed, LUS findings characterized by patchy bilateral SIS/white lung with or without small bilateral patchy subpleural consolidations can be compatible with COVID-19 pneumonia, although not definitively specific. Patients should be kept isolated and should quickly undergo both microbiological tests (both Sars-Cov-2 swab, intended as nasal/throat samples, and rapid serologic test for IgM antibodies) and high-resolution computed tomography scan of the chest (HRCT) with or without administration of iodinated contrast, depending on each clinical case and according to local protocols.

Patients should be hospitalized in isolation if:

1) At least one microbiological test is positive and/or

2) HRCT scan of the chest is suggestive for viral pneumonia

Patients could be hospitalized in general ward, according to local procedures, where a second swab will be performed, if:

1) Microbiological tests are negative and

2) HRCT scan of the chest is indicative of alternative diagnosis

Instead, if LUS is negative or showing findings not suggestive for a diffuse pneumogenic SIS, patients should be kept in isolation, because of the presence of suspected symptoms, waiting for results of microbiological tests (both nasal/ throat swab and rapid serologic tests).

At least one positivity of these tests indicates hospitalization in isolation. HRCT and/or contrast CT scan should be indicated in order to either rule out or rule in pulmonary lesions missed by LUS, especially in case of alteration of intrapulmonary gas exchanges $\left(\mathrm{paO}_{2} / \mathrm{FiO}_{2}<350\right.$ or change of usual, for chronic respiratory patients) detected by arterial blood gas (ABG) analysis.

Instead, if both microbiological test are negative, LUS not suggestive findings could foster an alternative diagnosis in combination with $\mathrm{ABG}$ analysis and panoramic $\mathrm{CXR}$ (which may add relevant information for pathologies not detectable by LUS):

1) LUS pattern suggestive of cardiogenic SIS: patients should undergo echocardiography to confirm diagnosis

2) LUS pattern and CXR pattern suggestive of alternative diagnosis (for example focal pneumonia, pleural effusions, mediastinal pathology, central lung consolidations etc.) with conserved intrapulmonary gas exchanges.

In these cases, patients could be hospitalized in general ward, according to local procedures, where a second swab will be performed.

However, as far as for the point 2 is concerned, an alteration of intrapulmonary gas exchanges $\left(\mathrm{paO}_{2} / \mathrm{FiO}_{2}<350\right.$ or change of usual, for chronic respiratory patients) should indicate HRCT and/or contrast CT scan in order to better explain the causes. Again, if CT scans are suspected for viral pneumonia, patients should be hospitalized in isolation, waiting for confirmation from other microbiologic tests (second swab test and serology for $\operatorname{IgG}, \operatorname{Ig} \mathrm{A} / \mathrm{M}$ antibodies).

Figure 2 shows the proposed algorithm to early manage patients with symptoms compatible with COVID-19 admitted to the emergency room.

\section{LUS and clinical management in pregnant women}

Pregnant women admitted to emergency room with symptomatology compatible with COVID-19 are managed in a dedicated COVID-19-path, hospitalized in isolation and should immediately receive LUS to define subsequent management paths. Pregnant women should have a normal LUS pattern, except for few rare particular cases. Sometimes, in advanced time of gestation, a mild posterior bilateral basal homogenous SIS is detectable for volume reduction of inferior lung lobes.

Therefore, if LUS findings are suggestive of patchy bilateral SIS with or without small bilateral patchy subpleural consolidations in a symptomatic context compatible with COVID-19, patients must keep isolation waiting for results of microbiological tests (both nasal/throat swab and rapid serologic tests). These LUS findings are highly suggestive of COVID-19 pneumonia, although not definitively specific. Indeed, LUS suggestive findings could foster therapeutic decisions [23, 24]. Patients need to be strictly monitored for worsening of symptoms and alteration of gas exchanges indicating ICU admission and wait for confirmation from other microbiologic tests (second 


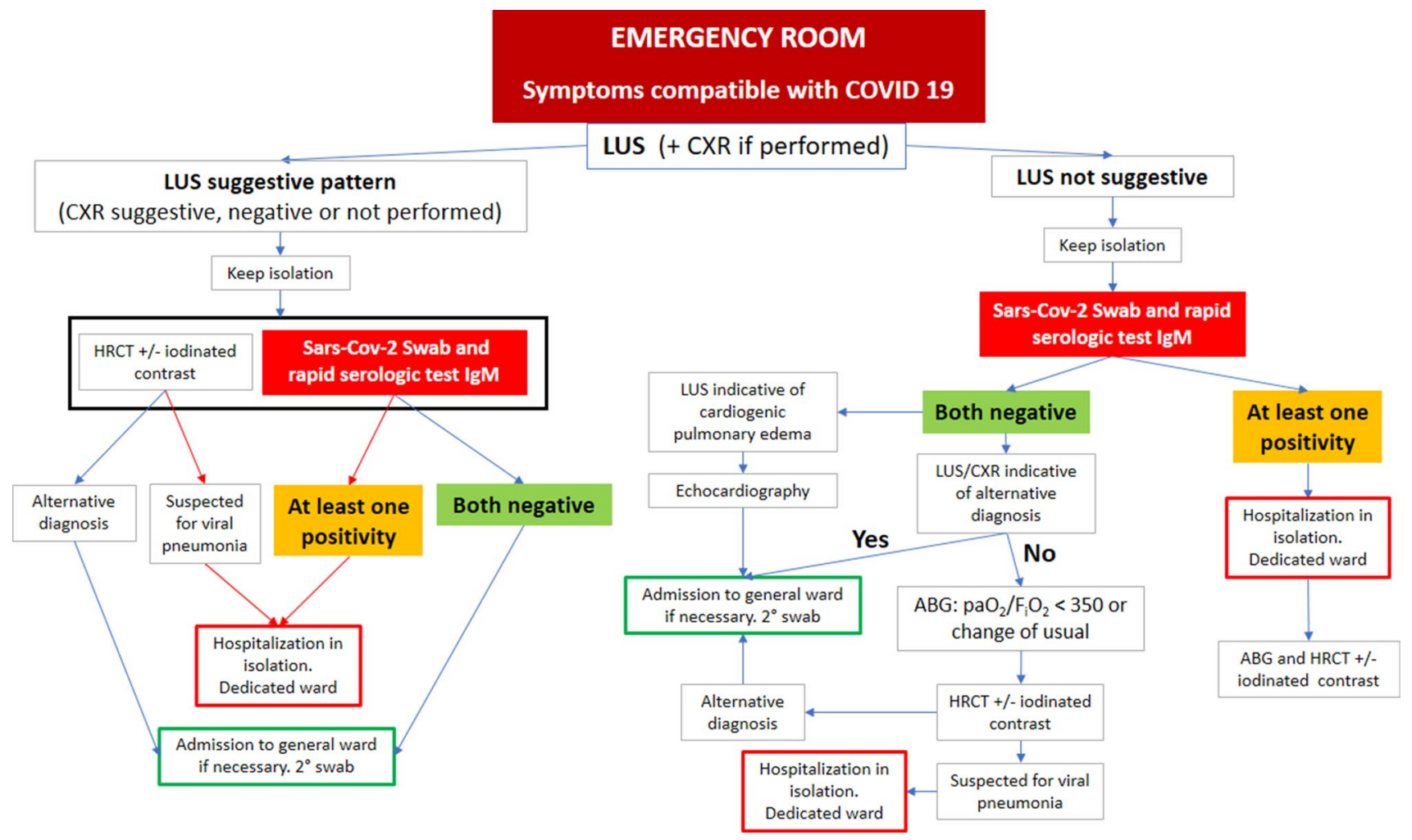

Fig. 2 A proposed algorithm to early manage patients with symptoms compatible with COVID-19 admitted to the emergency room

swab test and serology for $\mathrm{IgG}$, IgA/M antibodies) in case of fist microbiological tests both negative.

Instead, if LUS is not suggestive of suspicious patterns, it is important to focus on intrapulmonary gas exchanges detected by arterial blood gas (ABG) analysis. If $\mathrm{paO}_{2} /$ $\mathrm{FiO}_{2}$ ratio is altered in these young women $(<400)$, patients should be however kept in isolation, because of the presence of symptoms, waiting for microbiological tests (both nasal/throat swab and rapid serologic tests):

1) At least one positivity of these tests indicates hospitalization in isolations. It is very important to focus on worsening of symptoms, occurrence of relevant alteration of intrapulmonary gas exchanges $\left(\mathrm{paO}_{2} / \mathrm{FiO}_{2}<250\right)$ detected by arterial blood gas (ABG) analysis (a second LUS could show the appearance of a suggestive pattern). In these cases, ICU should be considered. In this context, gas exchanges data have to be considered with the final goal to achieve higher maternal oxygen (target arterial pressure of oxygen $\mathrm{paO}_{2}>70 \mathrm{mmHg}$ ) and lower carbon dioxide levels (target arterial pressure of carbon dioxide $\mathrm{paCO}_{2} 28-32 \mathrm{mmHg}$ ) to maintain placental perfusion and prevent fetal hypoxemia and acidosis. This is mandatory in pregnancy independently from the time of gestation [25].
2) In case of both negative results from microbiological tests: LUS could be indicative of an alternative diagnosis and a panoramic chest X-ray could add information useful to better stratify patients for alternative diagnosis not detectable by LUS (mediastinal pathology, focal consolidations not reaching visceral pleura etc.), while waiting for second Sars-Cov-2 swab results and admission to obstetric general ward.

In case of both LUS with no signs of suspected COVID19 pneumonia and normal intrapulmonary gas exchanges $\left(\mathrm{paO}_{2} / \mathrm{FiO}_{2}>400\right)$, pregnant women undergo maternal and fetal monitoring until microbiological test results.

At least one positivity requires that the patient should be kept in hospitalization in isolation.

If both microbiological tests are negative and $\mathrm{paO}_{2} /$ $\mathrm{FiO}_{2}>400$ the patient might be isolated at home and kept in contact either in case of worsening symptoms for hospital admission or in case of mild and stable symptoms for continuing home isolation waiting for the results of second Sars-Cov-2 swab test.

Figure 3 shows the proposed algorithm to early manage pregnant patients with symptoms compatible with COVID-19, admitted to the obstetric emergency room. 


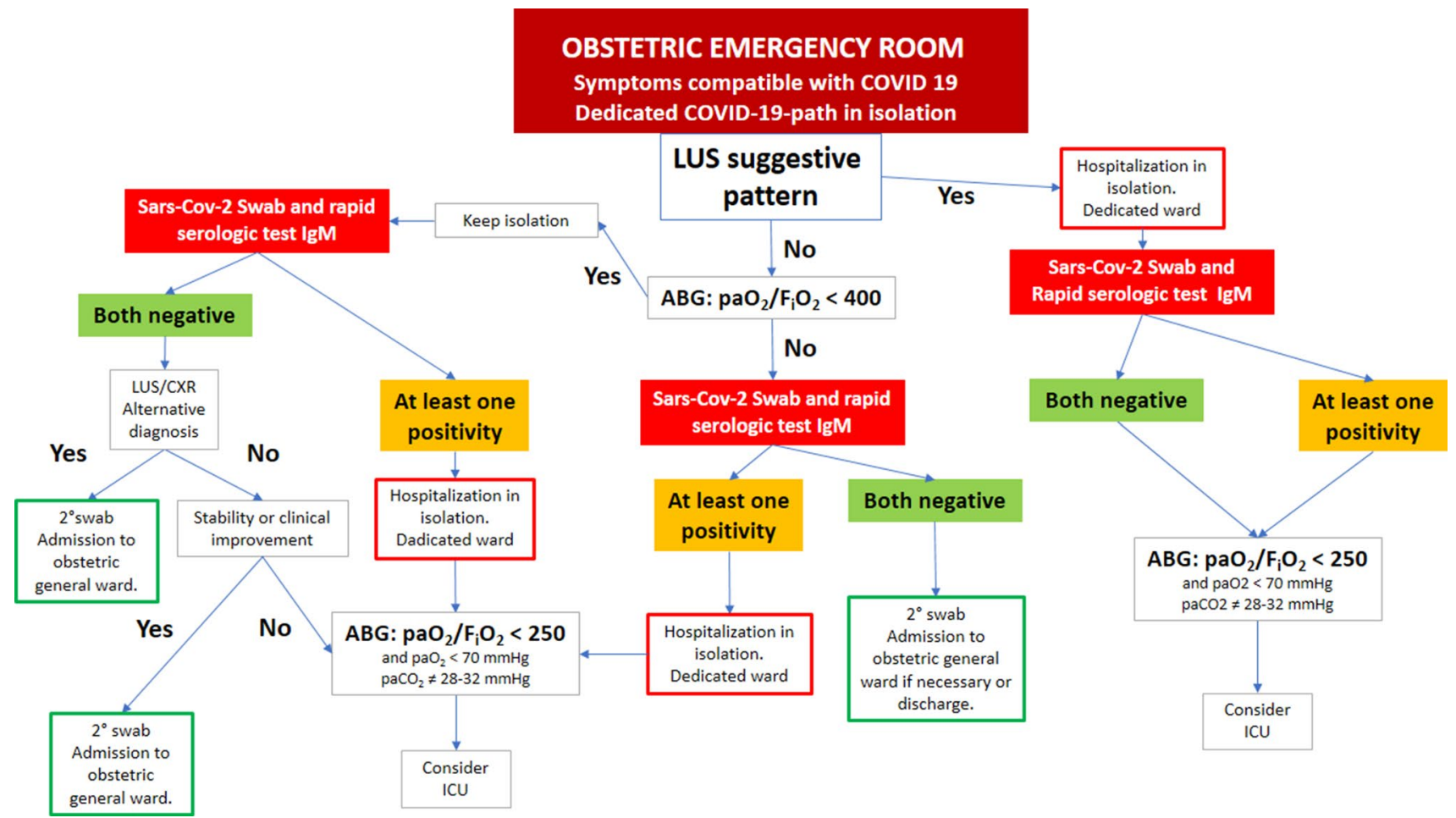

Fig. 3 A proposed algorithm to early manage pregnant patients with symptoms compatible with COVID-19, admitted to the obstetric emergency room

\section{LUS and clinical management in pediatric patients}

The role of LUS for pediatric patients has been already widely reported in literature [26-28]. In case of pediatric patients admitted to emergency room with symptomatology suspected for COVID-19, LUS should be early performed. As for pregnant woman, pediatric patients in basal conditions should have a normal LUS pattern. Therefore, a LUS pattern suggestive for pulmonary involvement in patients with symptomatology compatible with COVID-19 in a pandemic context, is highly indicative of viral pneumonia.

Patients should be kept hospitalized in isolation and strictly followed while waiting for results of microbiological tests (both nasal/throat swab and rapid serologic tests).

Instead, if LUS is not suggestive of suspicious patterns, it is important to focus on oxygen peripheral hemoglobin saturation $\left(\mathrm{SpO}_{2}\right)$. In presence of $\mathrm{SpO}_{2} \leq 95 \%$ or of need for $\mathrm{O} 2$ therapy, patients should be kept in isolation waiting for microbiological tests results:

1) At least one positivity of these tests indicates hospitalization in isolations. In case of worsening of symptoms or of gas exchanges with $\mathrm{SpO}_{2}<90 \%$ (a second LUS could show the appearance of a suggestive pattern), admission to pediatric ICU (PICU) and HRCT should be considered according to local protocols.
2) In case of both negative results from microbiological tests: LUS could be indicative of an alternative diagnosis and a panoramic chest X-ray could add information useful to better stratify patients for alternative diagnosis not detectable by LUS, while waiting for second Sars-Cov-2 swab results and admission to pediatric general ward.

The last clinical scenario is the one in which first LUS pattern is not compatible with COVID-19 pneumonia and there are normal values of $\mathrm{SpO}_{2}$. According to local protocols patients should undergo microbiological tests and could be isolated at home or hospitalized waiting for results.

Figure 4 shows the proposed algorithm to manage pediatric patients with symptoms compatible with COVID-19, admitted to the pediatric emergency room.

\section{Conclusions}

This paper aims to identify a role for LUS for early management of patients with symptoms suspected for COVID-19 admitted to emergency room in a pandemic context. Moreover, it tries to invite the medical community, operating in different specializations, to perform LUS with a standardized approach. Being able to recognize LUS patterns suggestive or not suggestive of COVID-19 pneumonia can be 


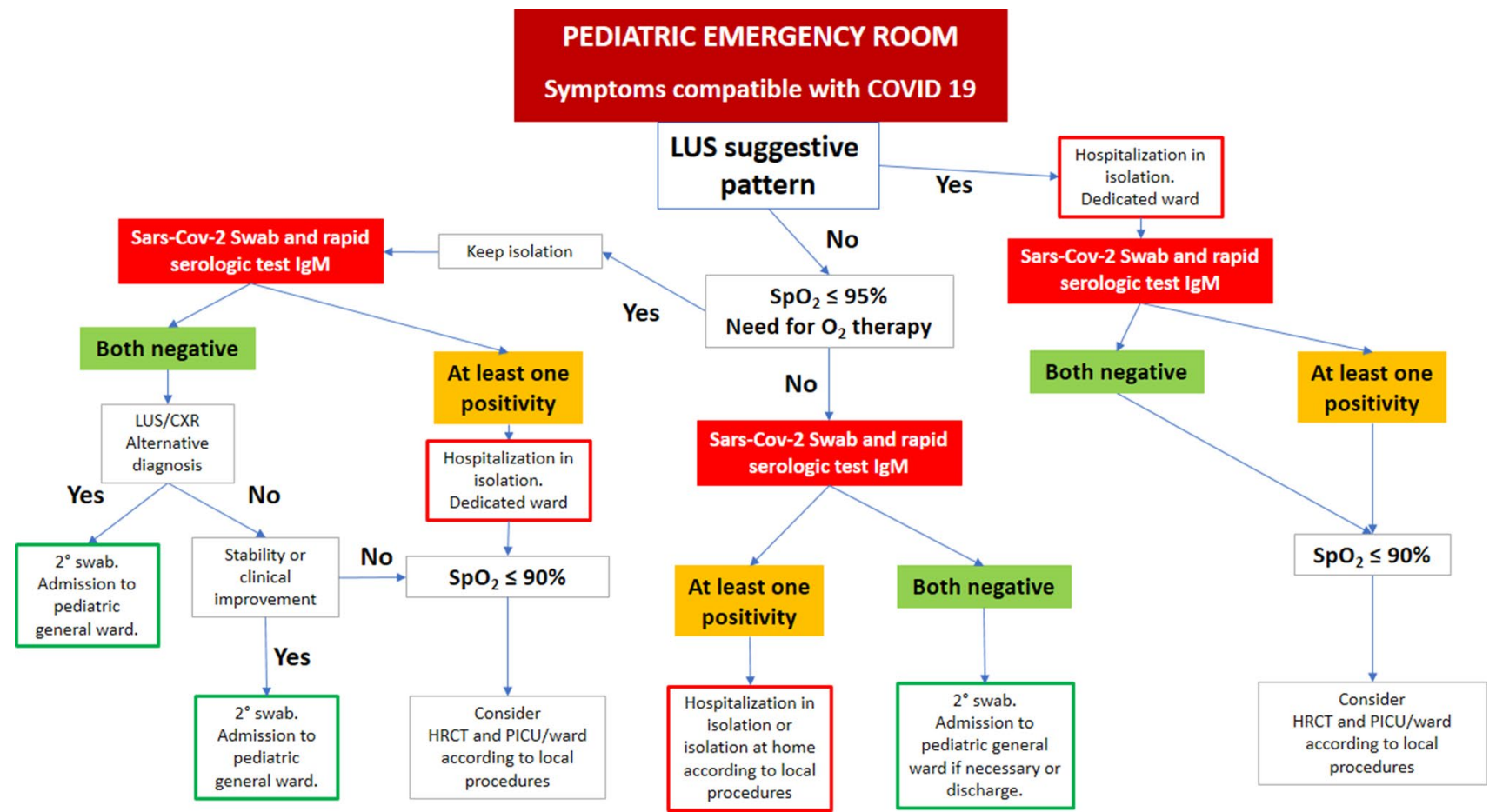

Fig. 4 A proposed algorithm to early manage pediatric patients with symptoms compatible with COVID-19, admitted to the pediatric emergency room

very useful and simplify diagnostic processes. Proposals of management algorithms are also reported to ameliorate daily clinical practice in a period of huge difficulties for healthcare workers in emergency department to face patients with suggestive but aspecific symptoms. Finally, we encourage also operators to collect echographic videos and images from COVID-19 patients and upload to a protected internationally available database allowing both the development and testing performances of automated algorithms dedicated to patterns recognition [29]. We invite our colleagues to link to our website (https://iclus-web.bluetensor.ai/ to upload images and videos to the database, to attend teaching programs and to test automated recognition of patterns). This should foster clinical cases discussion, comparison between physicians' evaluations and automated scores and educational programs of training in clinical ultrasonography.

Acknowledgements Authors would like to acknowledge Gemelli against COVID-19 group. Members are listed below:

Endocrinology: A. Bianchi, E. Capristo, A. Giampietro, A. Iaconelli, P. Locantore, D. Milardi, A. Pontecorvi. General Medicine: R. Cianci, G. De Matteis, F. De Vito, A. Porfidia, G. Gambassi. Geriatrics: F. Landi, A. Bellieni, V. Brandi, A. Carfî, E. D’Angelo, D. Fusco, G. Landi, R. Lo Monaco, A.M. Martone, E. Marzetti, F. Pagano, A. Russo, S. Salini, M. Tosato, A. Tummolo, R. Bernabei. Infectious Diseases: S. Di Giambenedetto, A. Borghetti, A. Ciccullo, A. Cingolani, K. de Gaetano Donati, A. Dusina, D. Faliero, M. Fantoni, D. Moschese, R. Murri, G. Scoppettuolo, E. Taddei, E. Tamburrini, G. Ventura, E. Visconti, R. Cauda. Internal Medicine and Gastroenterology: L. Zileri
Dal Verme, V. Abbate, S. Bibbò, F. D’Aversa, D. Feliciani, B. Funaro, M. Garcovich, M. Impagnatiello, L. Lopetuso, L. Miele, A. Nicoletti, A. Papa, M. Pizzoferrato, F. Pizzolante, M. Pompili, G. Rapaccini, F. Santopaolo, C. Settanni, A. Tosoni, A. Gasbarrini. Medical Clinic: F. Biscetti, A. Gallo, M. Montalto.

Authors' contributions All authors contributed to study conception and design. The first draft of the manuscript was written by Andrea Smargiassi and all authors commented on previous versions of the manuscript. Authors give all permissions to Journal of Ultrasound to publish this work. All authors read and approved the final manuscript

Funding Not applicable.

\section{Availability of data and material Yes.}

Code availability Not applicable.

Ethics approval Not applicable for review papers.

Conflict of interest None of the authors has to disclose conflicts of interest in relation to this work.

\section{References}

1. Tian S, Hu N, Lou J, Chen K, Kang X, Xiang Z, Chen H, Wang D, Liu N, Liu D, Chen G, Zhang Y, Li D, Li J, Lian H, Niu S, Zhang L, 
Zhang J (2020) Characteristics of COVID-19 infection in Beijing. J Infect 80(4):401-406

2. Chen J, Qi T, Liu L, Ling Y, Qian Z, Li T, Li F, Xu Q, Zhang Y, Xu S, Song Z, Zeng Y, Shen Y, Shi Y, Zhu T, Lu H (2020) Clinical progression of patients with COVID-19 in Shanghai, China. J Infect. https://doi.org/10.1016/j.jinf.2020.03.004 Epub ahead of print

3. Huang C, Wang Y, Li X, Ren L, Zhao J et al (2020) Clinical features of patients infected with novel 2019 coronavirus in Wuhan, China. Lancet 395:497-506

4. Zhang R, Ouyang H, Fu L, Wang S, Han J, Huang K, Jia M, Song Q, Fu Z (2020) CT features of SARS-CoV-2 pneumonia according to clinical presentation: a retrospective analysis of 120 consecutive patients from Wuhan city. Eur Radiol. https://doi.org/10.1007/s0033 0-020-06854-1

5. Peng QY, Wang XT, Zhang LN, Chinese Critical Care Ultrasound Study Group (CCUSG) (2020) Findings of lung ultrasonography of novel corona virus pneumonia during the 2019-2020 epidemic. Intensive Care Med. https://doi.org/10.1007/s00134-020-05996-6 (Epub ahead of print)

6. Soldati G, Smargiassi A, Inchingolo R, Buonsenso D, Perrone T, Federica D, Perlini S, Torri E, Mariani A, Mossolani EM, Demi L (2020) Is there a role for lung ultrasound during the COVID-19 outbreak? J Ultrasound Med. https://doi.org/10.1002/jum.15284 (Epub ahead of print)

7. Tian S, Hu W, Niu L, Liu H, Xu H, Xiao SY (2020) Pulmonary pathology of early-phase 2019 novel coronavirus (COVID-19) pneumonia in two patients with lung cancer. J Thorac Oncol pii S1556-0864(20):30132-30135

8. Soldati G, Giannesi G, Smargiassi A, Inchingolo R, Demi L (2020) COVID-19: pneumonia, ARDS or something else? J Ultrasound Med. https://doi.org/10.1002/jum.15338

9. Ciceri F, Beretta L, Scandroglio AM, Colombo S, Landoni G, Ruggeri A, Peccatori J, D’Angelo A, De Cobelli F, Rovere-Querini P, Tresoldi M, Dagna L, Zangrillo A. (2020) Microvascular COVID-19 lung vessels obstructive thromboinflammatory syndrome (MicroCLOTS): an atypical acute respiratory distress syndrome working hypothesis. Crit Care Resusc. [Epub ahead of print] PMID:32294809

10. Soldati G, Demi M, Smargiassi A, Inchingolo R, Demi L (2019) The role of ultrasound lung artifacts in the diagnosis of respiratory diseases. Expert Rev Respir Med 13:163-172

11. Soldati G, Smargiassi A, Inchingolo R, Sher S, Nenna R, Valente S, Inchingolo CD, Corbo GM (2015) Lung ultrasonography and vertical artifacts: the shape of air. Respiration. 90(1):86

12. Soldati G, Smargiassi A, Demi L, Inchingolo R (2020) Artifactual lung ultrasonography: it is a matter of traps, order, and disorder. Appl Sci. https://doi.org/10.3390/app10051570

13. Soldati G, Demi M, Inchingolo R, Smargiassi A, Demi L (2016) On the physical basis of pulmonary sonographic interstitial syndrome. J Ultrasound Med 35(10):2075-2086

14. Soldati G, Smargiassi A, Inchingolo R, Sher S, Nenna R, Valente S, Inchingolo CD, Corbo GM (2014) Lung ultrasonography may provide an indirect estimation of lung porosity and airspace geometry. Respiration 88(6):458-468

15. Demi M, Prediletto R, Soldati G, Demi L (2020) Physical mechanisms providing clinical information from ultrasound lung images: hypotheses and early confirmations. IEEE Trans Ultrason Ferroelectr Freq Control 67(3):612-623

16. Demi L, van Hoeve W, van Sloun RJG, Soldati G, Demi M (2017) Determination of a potential quantitative measure of the state of the lung using lung ultrasound spectroscopy. Sci Rep 7(1):12746

17. Soldati G, Smargiassi A, Inchingolo R, Buonsenso D, Perrone T, Briganti DF, Perlini S, Torri E, Mariani AA, Mossolani EE, Tursi F, Mento F, Demi L (2020) Proposal for international standardization of the use of lung ultrasound for COVID-19 patients; a simple, quantitative, reproducible method. J Ultrasound Med. https://doi. org/10.1002/jum.15285 (Epub ahead of print)
18. Kampf G, Todt D, Pfaender S, Steinmann E (2020) Persistence of coronaviruses on inanimate surfaces and their inactivation with biocidal agents. J Hosp Infect 104(3):246-251. https://doi.org/10.1016/j. jhin.2020.01.022

19. Copetti R, Soldati G, Copetti P (2008) Chest sonography: a useful tool to differentiate acute cardiogenic pulmonary edema from acute respiratory distress syndrome. Cardiovasc Ultrasound 29(6):16

20. Sofia S, Boccatonda A, Montanari M et al (2020) Thoracic ultrasound and SARS-COVID-19: a pictorial essay. J Ultrasound 23:217-221

21. Moro F, Buonsenso D, Moruzzi MC, Inchingolo R, Smargiassi A, Demi L, Larici AR, Scambia G, Lanzone A, Testa AC (2020) How to perform lung ultrasound in pregnant women with suspected COVID-19 infection. Ultrasound Obstet Gynecol. https:// doi.org/10.1002/uog.22028

22. Buonsenso D, Moro F, Inchingolo R, Smargiassi A, Demi L, Soldati G, Moroni R, Lanzone A, Scambia G, Testa AC (2020) Effectiveness of a 'fast lung ultrasound teaching program' for gynecologists/obstetricians dealing with pregnant women with suspicion of COVID-19 infection. Ultrasound Obstet Gynecol. https://doi. org/10.1002/uog.22066 (Epub ahead of print)

23. Buonsenso D, Raffaelli F, Tamburrini E, Biasucci DG, Salvi S, Smargiassi A, Inchingolo R, Scambia G, Lanzone A, Testa AC, Moro F (2020) Clinical role of lung ultrasound for the diagnosis and monitoring of COVID-19 pneumonia in pregnant women. Ultrasound Obstet Gynecol. https://doi.org/10.1002/uog.22055

24. Inchingolo R, Smargiassi A, Moro F, Buonsenso D, Salvi S, Del Giacomo P, Scoppettuolo G, Demi L, Soldati G, Testa AC (2020) The diagnosis of pneumonia in a pregnant woman with covid-19 using maternal lung ultrasound. Am J Obstet Gynecol pii S00029378(20):30468-3. https://doi.org/10.1016/j.ajog.2020.04.020

25. Dharani K, Narendra DM, Kalpalatha KG (2019) Acute respiratory distress syndrome in pregnancy. In: Jeffrey P, Phelan LDP, Foley MR, Saade GR, Dildy GA, Belfort MA (eds) Critical care obstetrics. Wiley-Blackwell, New Jersey, pp 403-418

26. Buonsenso D, Piano A, Raffaelli F, Bonadia N, de Gaetano Donati K, Franceschi F (2020) Point-of-Care Lung Ultrasound findings in novel coronavirus disease-19 pnemoniae: a case report and potential applications during COVID-19 outbreak. Eur Rev Med Pharmacol Sci 24(5):2776-2780

27. Xin H, Li J, Hu HY (2018) Is lung ultrasound useful for diagnosing pneumonia in children? A meta-analysis and systematic review. Ultrasound Q 34(1):3-10

28. De Rose $\mathrm{C}$, Inchingolo $\mathrm{R}$, Smargiassi A, Zampino G, Valentini $\mathrm{P}$, Buonsenso D (2020) How to perform pediatric lung ultrasound examinations in the time of COVID-19. J Ultrasound Med. https:// doi.org/10.1002/jum.15306 (Epub ahead of print)

29. Roy S, Menapace W, Oei S, Luijten B, Fini E, Saltori C, Huijben I, Chennakeshava N, Mento F, Sentelli A, Peschiera E, Trevisan R, Maschietto G, Torri E, Inchingolo R, Smargiassi A, Soldati G, Rota P, Passerini A, Van Sloun RJG, Ricci E, Demi L (2020) Deep learning for classification and localization of COVID-19 markers in point-of-care lung ultrasound. IEEE Trans Med Imaging. https://doi. org/10.1109/TMI.2020.2994459

Publisher's Note Springer Nature remains neutral with regard to jurisdictional claims in published maps and institutional affiliations. 